\title{
Effect of Continuous Positive Airway Pressure on Chronic Cough in Obstructive Sleep Apnea Concomitant Gastroesophageal Reflux Patients: A Retrospective Observational Study
}

Jiao Su

Zhengzhou University First Affiliated Hospital https://orcid.org/0000-0001-6925-9430

Chunling Zhao

Zhengzhou University First Affiliated Hospital

\section{Yang Meng}

Zhengzhou University First Affiliated Hospital

\section{Yifei Fang}

Zhengzhou University First Affiliated Hospital

\section{Yanjun Liu}

Zhengzhou University First Affiliated Hospital

\section{Linge Sun}

Zhengzhou University First Affiliated Hospital

\section{Mengge Wang}

Zhengzhou University First Affiliated Hospital

\section{Liping Dai}

Zhengzhou University

\section{Songyun Ouyang ( $\nabla$ ouyangsy@163.com )}

First Affiliated Hospital of Zhengzhou University https://orcid.org/0000-0003-1575-027X

\section{Research}

Keywords: chronic cough, gastroesophageal reflux, obstructive sleep apnea, continuous positive airway pressure

Posted Date: July 14th, 2021

DOI: https://doi.org/10.21203/rs.3.rs-694754/v1

License: (c) (i) This work is licensed under a Creative Commons Attribution 4.0 International License. Read Full License 


\section{Abstract}

\section{Background:}

Chronic cough is common in obstructive sleep apnea. The aetiology of chronic cough in obstructive sleep apnea patients could be multifactorial. Several studies have reported high incidence rates of gastroesophageal reflux in obstructive sleep apnea patients, and gastroesophageal reflux may be one of causes of chronic cough in OSA patients. This study aimed to investigate the effect of continuous positive airway pressure on chronic cough in obstructive sleep apnea concomitant gastroesophageal reflux patients.

\section{Methods:}

Obstructive sleep apnea concomitant gastroesophageal reflux patients who also suffered chronic cough were enrolled. Patients who accepted continuous positive airway pressure as treatment group, and the patients who accepted general treatment (diet control, moderate exercise, sleeping on your side, limiting alcohol consumption and proton pump inhibitor \pm mosapride) as control group. Effects of different treatment modalities on chronic cough were assessed, and the correlation between chronic cough, obstructive sleep apnea and gastroesophageal reflux were performed by Pearson correlation analyses.

\section{Results:}

All Patients had poor sleep quality, severe daytime sleepiness, moderate-severe sleep apnea, significant weak acid reflux and moderate coughing events at the baseline. Compared with the baseline, PSQI and ESS score were improved in treatment group after 1 week's treatment, the improvement became stronger with duration of therapy ( $P \otimes 0.05)$. GERD-Q score, VAS score, daytime and night cough symptom score were all significantly improved in these two groups after treatment $(P \otimes 0.05)$, while this improvement was more pronounced in the treatment group ( $P \otimes 0.05$ ). There were significant correlation between $\mathrm{AHI}$ and VAS score $(\mathrm{r}=0.612, P 20.05)$, weak acid reflux and VAS score $(\mathrm{r}=0.429, P \& 0.05)$, weak acid reflux and AHI $(\mathrm{r}=0.366, P 20.05)$.

\section{Conclusion:}

The improvement in chronic cough and gastroesophageal reflux was due to the resolution of obstructive sleep apnea. AHI and weak acid reflux may be critical factors affecting the therapeutic effect of chronic cough in obstructive sleep apnea concomitant gastroesophageal reflux patients.

\section{Background}

Chronic cough, which affects $2.3-33 \%$ of the adult population ${ }^{[1,2]}$, is one of the most common complaints in respiratory outpatient clinic visits. A significant fraction of chronic cough remains undiagnosed, and the percentage of unexplained cough has ranged up to $42 \%{ }^{[3]}$. Obstructive sleep apnea (OSA) is being increasingly recognized as cause of chronic cough. The aetiology of chronic cough in OSA 
patients could be multifactorial. The incidence of gastroesophageal reflux (GER) is high in OSA patients, and GER may be one of the causes of chronic cough in OSA patients.

OSA is characterized by repetitive collapse of the upper airway during sleep, with the development of larger negative intrathoracic pressures during inspiratory efforts against the occluded airway. Recent evidence points to a link between chronic cough and OSA. OSA is being increasingly recognized as cause of chronic cough ${ }^{[4-6]}$. Similar to OSA, GER which can co-exist or not in the same individual, is a common cause of chronic cough. The prevalence of GER was significantly higher in OSA patients than general population $^{[6-8]}$. Gastroesophageal reflux (GER) may be one of the causes of chronic cough in OSA patients. Previous study have concluded that proton pump inhibitor (PPI) therapy for GER can not improve cough in GER patients ${ }^{[9]}$. Continuous positive airway pressure (CPAP) has been shown to improve GER events in OSA patients ${ }^{[10]}$. Unfortunately, as far as OSA and GER-related cough is concerned, the therapeutic management remains largely empirical. This study aimed to determine the effect of different treatment modalities on chronic cough in OSA concomitant GER patients, and the possible mechanism of these association.

\section{Methods}

\section{Subjects}

This study was a retrospective observational study. Adult patients with chronic cough seen at Department of Respiratory and Sleep Medicine of First affiliated Hospital of Zhengzhou University were given the option to enroll into this study between Mar. 2017 and Nov. 2020. This study was carried out in accordance with the WMA Declaration of Helsinki, and approved by the grass-roots ethics committee of First affiliated Hospital of Zhengzhou University and the ethics committee of Zhengzhou University.

Exclusion criteria: (1) Any other diseases that resulted in chronic cough. (2) Chronic disease states such as congestive heart failure, chronic kidney disease, cancer, need for immunosuppressive therapy, or diabetes mellitus. (3) History of hypertension, and poor blood pressure control.

Inclusion criteria: (1) Cough more than 8 weeks. (2) Normal spirometry and provocation test. (3) Normal chest radiographs and/or CT scans of the chest. (4) Normal acidophilic granulocyte count of induced sputum. (5) Normal skin phadiatop test. (6) Normal paranasal sinus computed tomography. (7) No history of ACEl drugs.

\section{Pittsburgh Sleep Quality Index (PSQI)}

Sleep quality was assessed using the Chinese version of the PSQI ${ }^{[11]}$. The PSQI is a self-rated questionnaire that can assess sleep quality and sleep disturbances over one-month period. It consists of 18 items which are divided into 7 aspects: sleep quality, sleep latency, sleep duration, sleep efficiency, sleep disturbance, use of sleep medication, and daytime dysfunction. Each answer is graded on a fourpoint scale ranging from 0 to 3 . The score ranges from 0 to 21 . Higher PSQI score, poorer sleep quality. 


\section{Epworth sleepiness scale (ESS)}

Daytime sleepiness was assessed using the Chinese version of the ESS ${ }^{[12]}$. It consists of eight questions regarding how often an individual dozes during daily activities. Each answer is graded on a four-point scale ranging from 0 to 3.The ESS score ranges from 0 (never dozed) to 24 (high chance of dozing). Higher ESS score, greater sleep propensity during the day.

\section{GERD - Questionnaire (GERD-Q)}

Gastroesophageal reflux symptom was evaluated using the Chinese version of GERD-Q [13]. It consists of six questions which are divided into three aspects: heartburn and reflux, epigastric pain and nausea, sleep disorder and PPI use within the past one week. The total score of 8 or greater indicates GERD.

\section{Visual Analogue Scale (VAS)}

VAS for cough ${ }^{[14]}$ is used for assess the severity of cough by linear integral method. It ranges from 0 to 10. 0 indicates asymptomatic, and 10 indicates the most severity of cough. It can be used for the longitudinal comparison before and after the treatment.

\section{Cough symptom score}

Daytime and nighttime cough symptom was assessed using Chinese version of cough symptom score according to the 2015 guideline for the diagnosis and treatment of cough in China ${ }^{[14]}$. This scale is divided into 2 parts, daytime and nighttime cough symptom scores. The score ranges 0 to 3 , with 0 being no cough and 3 being the most severe cough.

\section{4h esophageal Multichannel Intraluminal Impedance-pH (24h MII-pH)}

- 24h MIl-pH (Medtronic, Inc. USA) monitoring was used to diagnose GER. Patients were not treated with antiacid during the examination or within 8 weeks before the examination. Abnormal GER was defined when the DeMeester score $\geq 14.72^{[15]}$ and/or meet the criteria of Lyon Consensus ${ }^{[16]}$.

\section{Polysomnography}

Overnight polysomnography monitoring were performed using Compumedics systems. Categorisation of sleep stage and respiratory events were performed according to American Academy of Sleep Medicine criteria (AASM Version 2.4) ${ }^{[17]}$. The severity of sleep disordered breathing was defined by the apneahypopnea index $(\mathrm{AHI})$.

24h MII-pH (Medtronic, Inc. USA) combined with polysomnography monitoring was performed for each patient. A synchronisation signal of $\mathrm{pH}$ was recorded in both the reflux system and the Compumedics system to allow accurate analysis of the temporal relationship between apnea and reflux events. 


\section{Protocol}

Based on the above inclusion-exclusion criteria, patients with chronic cough were enrolled. After relevant auxiliary examinations, OSA concomitant GER patients were further collected. General treatment includes diet control, moderate exercise, sleeping on your side, limiting alcohol consumption and medications (PPI \pm mosapride). According to the patient preference and compliance, the patients were divided into two groups, treatment group (CPAP + general treatment) and control group (general treatment). The study protocol is as follows (Fig. 1).

\section{Statistical analysis}

Results are presented as mean \pm SD. For the analysis of PSQI, ESS, GERD-Q, VAS, cough symptom score between treatment group and control group, we performed a repeated measures multivariate analysis of variance (MANOVA). For the analysis of AHI, the longest time of apnea, the lowest oxygen saturation, Demeester score, acid reflux, weak acid reflux and non-acid reflux between treatment group and control group, we performed independent sample t-test. Pearson correlation coefficients were used to examine the correlation between variables and chronic cough. $P<0.05$ was considered statistically significant. All statistical analysis was performed in SPSS 16.0.

\section{Results}

\section{Baseline characteristics}

Out of a total of 86 patients who meet the criteria were enrolled into the study between Mar. 2017 and Nov. 2020, 40 patients accepted general treatment as control group and 46 patients accepted CPAP as treatment group. The baseline data of this study are shown in Table 1. Patients in both groups had poor sleep quality, severe daytime sleepiness, sleep structural disorder, decreased sleep efficiency, moderatesevere sleep apnea, significant weak acid reflux, and moderate coughing events. These parameters were no statistical difference between control group and treatment groups at the baseline. 
Table 1

The baseline data of this study.

\begin{tabular}{|c|c|c|}
\hline & Treatment group $(n=46)$ & Control group $(n=40)$ \\
\hline Age & $36.91 \pm 5.55$ & $37.52 \pm 5.92$ \\
\hline $\operatorname{Sex}(M / F)$ & $26 / 20$ & $24 / 16$ \\
\hline $\operatorname{BMI}\left(\mathrm{kg} / \mathrm{m}^{2}\right)$ & $27.48 \pm 4.75$ & $28.38 \pm 4.94$ \\
\hline \multicolumn{3}{|l|}{ Smoking: } \\
\hline Yes & 26 & 25 \\
\hline No & 20 & 15 \\
\hline \multicolumn{3}{|l|}{ Drinking: } \\
\hline Yes & 28 & 27 \\
\hline No & 18 & 13 \\
\hline PSQI & $12.09 \pm 2.49$ & $11.63 \pm 2.36$ \\
\hline ESS & $14.33 \pm 2.46$ & $14.08 \pm 2.28$ \\
\hline GERD-Q & $4.09 \pm 2.98$ & $4.43 \pm 2.51$ \\
\hline $\mathrm{TST} / \mathrm{min}$ & $398.33 \pm 23.03$ & $404.18 \pm 23.15$ \\
\hline Sleep efficiency(\%) & $73.15 \pm 5.59$ & $75.18 \pm 4.47$ \\
\hline \multicolumn{3}{|l|}{ Sleep structure: } \\
\hline N1(\%) & $15.04 \pm 2.09$ & $13.15 \pm 2.52$ \\
\hline N2(\%) & $68.28 \pm 5.00$ & $70.53 \pm 2.14$ \\
\hline N3(\%) & $8.56 \pm 2.51$ & $10.05 \pm 2.01$ \\
\hline $\mathrm{R}(\%)$ & $7.89 \pm 3.02$ & $6.03 \pm 3.07$ \\
\hline $\mathrm{AHI}(/ \mathrm{h})$ & $30.24 \pm 5.45$ & $29.73 \pm 4.03$ \\
\hline Longest time of apnea(min) & $43.12 \pm 8.29$ & $41.26 \pm 4.60$ \\
\hline $\mathrm{LSaO}_{2}(\%)$ & $81.09 \pm 3.33$ & $80.08 \pm 4.35$ \\
\hline Demeester score & $18.23 \pm 7.77$ & $17.71 \pm 5.15$ \\
\hline Acid reflux & $29.33 \pm 11.34$ & $27.77 \pm 13.84$ \\
\hline Weak acid reflux & $24.60 \pm 9.14$ & $23.89 \pm 7.95$ \\
\hline Non-acid reflux & $2.14 \pm 1.30$ & $1.12 \pm 1.09$ \\
\hline
\end{tabular}




\begin{tabular}{|lll|}
\hline & Treatment group $(n=46)$ & Control group $(n=40)$ \\
\hline VAS & $55.76 \pm 10.59$ & $52.38 \pm 8.09$ \\
\hline Daytime cough symptom & $2.13 \pm 0.81$ & $2.03 \pm 0.80$ \\
\hline Nighttime cough symptom & $1.83 \pm 0.77$ & $1.73 \pm 0.82$ \\
\hline
\end{tabular}

Compared with the baseline, PSQI (Fig. 2a) and ESS (Fig. 2b) were improved in treatment group after 1 week treatment, and the improvement became stronger with duration of therapy (P囚0.05). GERD-Q (Fig. 3), VAS (Fig. 4a), daytime (Fig. 4b) and night cough symptom score (Fig. 4c) were all significantly improved after treatment $(P \otimes 0.05)$, the improvement were more pronounced in the treatment group (P®0.05). Furthermore, there were significant correlation between AHI and VAS (Fig. 5a, $r=0.612, P \unrhd 0.05$ ), weak acid reflux and VAS (Fig. 5b, $r=0.429, P \llbracket 0.05$ ). Weak acid reflux was significantly correlated with AHI (Fig. 5c, $r=0.366, P \bowtie 0.05)$.

\section{Discussion}

The major findings of this study were as follows: (1) CPAP improved symptoms of chronic cough and GER in OSA concomitant GER patients. (2) AHI and weak acid reflux were both significantly correlated with chronic cough, AHI was also correlated with weak acid reflux. This findings are consistent with other studies that CPAP treatment can improve $\operatorname{GER}^{[10,18]}$ and daytime sleepiness ${ }^{[10]}$. Previous studies investigated the effect of CPAP on GER and OSA, but there had been no analysis focus on the effect of CPAP on chronic cough in OSA concomitant GER patients. The present study is the first to explore the effects of different treatment modalities on chronic cough in OSA concomitant GER patients.

OSA and GER are being increasingly recognized as causes of chronic cough. Previous study found that GER symptom was improved after 1 week's CPAP treatment in OSA concomitant GER patients ${ }^{[18]}$. Tamanna et al. further found that GER and daytime sleepiness were improved after 6 months' CPAP treatment in OSA concomitant GER patients ${ }^{[10]}$. However, no studies have yet confirmed the efficacy of CPAP on chronic cough in OSA concomitant GER patients. Thus, 86 OSA concomitant GER patients who also suffered from chronic cough were collected in present study. We assessed the association between cough, GER, and treatment response in OSA concomitant GER patients and found that the sleep quality, daytime sleepiness, symptoms of cough and GER were all markedly improved after 3 months' CPAP treatment in these patients. Previous study suggested that PPI can not improve cough symptom in GER patients, while lifestyle modifications and weight loss may be beneficial for chronic cough in GER patients $^{[9]}$. In our study, we further found that general treatment and PPI improved the symptoms of cough and GER, while the improvement was more pronounced after CPAP treatment. Unfortunately, general treatment and medications had no effect on sleep quality and daytime sleepiness in OSA concomitant GER patients. 
- The 24h MIl-pH monitoring can accurately distinguish and analyze forms of reflux, such as acid reflux, weak acid reflux, and non-acid reflux ${ }^{[19]}$. OSA concomitant GER patients were diagnosed using 24h MIl-pH combined with overnight PSG monitoring in present study. We discovered an association between AHI and VAS, weak acid reflux and VAS, AHI and weak acid. These findings are consistent with several, but not all, previous studies ${ }^{[8,20,21]}$. Previous studies have reported the relationship between $\mathrm{AHI}$ and GER using symptoms ${ }^{[6]}$, responses to anti-GER medication ${ }^{[22]}, 24 \mathrm{~h} \mathrm{pH}$ monitoring ${ }^{[22]}$ or endoscopic finding ${ }^{[8]}$ to define GER, rather than $24 \mathrm{~h} \mathrm{MII-pH}$. The symptoms of GER, such as weak acid reflux, may have been masked by the severity of the cough and not considered by the physician. With $24 \mathrm{~h} \mathrm{MII-pH}$ monitoring is widely used, clinicians find that not all forms of reflux can cause symptoms in GER patients. The diagnostic yield of $24 \mathrm{~h}$ esophageal $\mathrm{pH}$ monitoring in patients with extra-esophageal symptoms is probably far from perfect. Previous study has found that the frequency of weak acid reflux is significantly higher in GER patients with cough than in patients without cough ${ }^{[23]}$. It probable reveal why some reflux episodes in the GER patient causes cough while others do not. Our study indicates that both AHI and weak acid reflux may play important roles in the the pathogenesis of chronic in OSA concomitant GER patients.

Several studies have suggested that OSA causes GER, rather than the reverse, while there was no objective parameters to assess $A H I$ and reflux episodes in these studies ${ }^{[24,25]}$. The mechanism by which CPAP reduces the symptom of chronic cough in OSA concomitant GER patients is not fully understood. Several studies have demonstrated that CPAP can make lower esophageal sphincter (LES) less susceptible to reflux by increasing barrier pressure to reflux and decreasing the duration of LES relaxation ${ }^{[26,27]}$. We speculated that the probable mechanism of cough resolution with CPAP is through the improvement of upper airway collapse, further promoting the barrier function of LES, and then alleviating weak acid reflux in OSA patients.

The limitations of the present study are as follows. First, there was no blank control group. We believed that it would not be ethical to withhold therapy from patients. Second, the subjective parameters such as sleep quality, daytime sleepiness, GER related questionnaire and cough related questionnaires could be influenced by patients' judgment.These questionnaires are simple and practicable to perform, and there is no objective cough-related monitoring up to now. Moreover, professional technicians operate these questionnaires strictly and accurately according to the instructions in our study. Third, our study is a retrospective observational study, further fundamental research and large-scale prospective studies are needed to draw a more definitive conclusion.

\section{Conclusions}

In summary, this results indicates that the improvement in chronic cough and GER was due to the resolution of OSA. AHI and weak acid reflux may be critical factors affecting the therapeutic effect of chronic cough in OSA concomitant GER patients. We speculated that the probable mechanism of cough resolution with CPAP is through the improvement of upper airway collapse, further promoting the barrier 
function of LES, and then alleviating weak acid reflux in OSA patients. Further fundamental research and large-scale prospective studies are needed to draw a more definitive conclusion in the future.

\section{Abbreviations}

obstructive sleep apnea OSA

gastroesophageal reflux GER

proton pump inhibitor PPI

continuous positive airway pressure CPAP

apnea-hypopnea indices AHI

Pittsburgh Sleep Quality Index PSQI

Epworth sleepiness scale ESS

GERD - Questionnaire GERD-Q

Visual Analogue Scale VAS

24h esophageal Multichannel Intraluminal Impedance-pH 24h MII-pH

repeated measures multivariate analysis of variance MANOVA

lower esophageal sphincter LES

\section{Declarations}

\section{Ethics approval and consent to participate}

This study was carried out in accordance with the WMA Declaration of Helsinki, and approved by the grass-roots ethics committee of First affiliated Hospital of Zhengzhou University and the ethics committee of Zhengzhou University.

\section{Consent for publication}

Not applicable

\section{Availability of data and materials}

The datasets used and/or analysed during the current study are available from the corresponding author on reasonable request. 


\section{Competing interests}

The authors declare that they have no competing interests.

\section{Funding}

Supported by grant No. U1804195 from National Natural Science Foundation of China.

\section{Authors' contributions}

JS participated in the design of the study, analyzed data, interpreted data and drafted manuscript. CZ, YM and YF carried out the study. YL, LS, and MW analyzed data, drafted manuscript. LD completed statistical analysis. SO conceived of the study, participated in the design of the study, edited and revised manuscript. All authors read and approved the final manuscript.

\section{Acknowledgements}

Not applicable.

\section{References}

1. Chung KF, Pavord ID. Chronic cough prevalence, pathogenesis, and causes of chronic cough. Lancet. 2008;371(9621):1364-74.

2. Song WJ, Chang YS, Faruqi S, Kim JY, Kang MG, Kim S, et al. The global epidemiology of chronic cough in adults: a systematic review and meta-analysis. Eur Respir J. 2015;45(5):1479-81.

3. McGarvey LP. Does idiopathic cough exist? Lung. 2008;186(suppl 1):78-81.

4. Chan KK, Ing AJ, Laks L, Cossa G, Rogers P, Birring SS. Chronic cough in patients with sleepdisordered breathing. Eur Respir J. 2010;35(2):368-72.

5. Shi C, Liang S, Xu X, Chen Q, Wang L, Yu L, et al. Cough hypersensitivity in patients with obstructive sleep apnea hypopnea syndrome. Sleep Breath. 2019;23(1):33-9.

6. Gouveia CJ, Yalamanchili A, Ghadersohi S, Price CPE, Bove M, Attarian PH, et al. Are chronic cough and laryngopharyngeal reflux more common in obstructive sleep apnea patients? Laryngoscope. 2019;129(5):1244-9.

7. Sabate JM, Jouet P, Merrouche M, Pouzoulet J, Maillard D, Harnois F, et al. Gastroesophageal reflux in patients with morbid obesity: a role of obstructive sleep apnea syndrome? Obes Surg. 2008;18(11):1479-84.

8. Kim Y, Lee YJ, Park JS, Cho YJ, Yoon HI, Lee JH, et al. Associations between obstructive sleep apnea severity and endoscopically proven gastroesophageal reflux disease. Sleep Breath. 2018;22(1):8590.

9. Kahrilas P, Altman KW, Chang AB. Chronic Cough Due to Gastroesophageal Reflux in Adults: CHEST Guideline and Expert Panel Report. Chest. 2016;150(6):1341-60. 
10. Tamanna S, Campbell D, Warren R, Ullah MI. Effect of CPAP therapy on symptoms of nocturnal gastroesophageal reflux among patients with obstructive sleep apnea. J Clin Sleep Med. 2016;12(9):1257-61.

11. Mollayeva T, Thurairajah P, Burton K, Mollayeva S, Shapiro CM, Colantonio A.

12. The Pittsburgh sleep. quality index as a screening tool for sleep dysfunction in clinical and nonclinical samples: A systematic review and meta-analysis. Sleep Med Rev. 2016;25:52-73.

13. Johns MW. A new method for measuring daytime sleepiness: the Epworth sleepiness scale. Sleep. $1991 ; 14(6): 540-5$.

14. Teklu M, Gouveia CJ, Yalamanchili A, Ghadersohi S, Price CPE, Bove M, et al. Predicting Obstructive Sleep Apnea Status With the Reflux Symptom Index in a Sleep Study Population. Laryngoscope. 2020; 130 (12): E952 - E957.

15. The asthma group of Chinese Thoracic Society. guideline for the diagnosis and treatment of cough (2015). Chin J Tuberc Respir Dis. 2016;39(5):323-54.

16. Neto RML, Herbella FAM, Schlottmann F, Patti MG. Does DeMeester score still define GERD? Dis Esophagus. 2019; 1;32(5):doy118.

17. Gyawali CP, Kahrilas PJ, Savarino E, Zerbib F, Mion F, Smout AJPM, et al. Modern diagnosis of GERD: the Lyon Consensus. Gut. 2018;67(7):1351-62.

18. Berry RB, Brooks Rita, Gamaldo CE, et al, for the American Academy of Sleep Medicine. The AASM Manual for the Scoring of Sleep and Associated Events: Rules, Terminology, and Technical Specifications. Version 2.4. Darien: American Academy of Sleep Medicine; 2017.

19. Tawk M, Goodrich S, Kinasewitz G, Orr W. The effect of 1 week of continuous positive airway pressure treatment in obstructive sleep apnea patients with concomitant gastroesophageal reflux. Chest. 2006;130(4):1003-8.

20. Boeckxstaens GE,Smout A.Systematic review: ro1e of acid,weakly acidic and.

21. Weakly alkaline reflux ingastro-oesophageal reflux disease.Aliment Pharmacol Ther.

22. 2010.; 32:332-343.

23. Vela MF, Kramer JR, Richardson PA, Dodge R, El-Serag HB. Poor sleep quality and obstructive sleep apnea in patients with GERD and Barrett's esophagus. Neurogastroenterol Motil. 2014;26(3):346-52.

24. Ju G, Yoon I, Lee SD, Kim N. Relationships between sleep disturbances and gastroesophageal reflux disease in Asian sleep clinic referrals. J Psychosom Res. 2013;75(6):551-5.

25. Faruqi S, Fahim A, Morice AH. Chronic cough and obstructive sleep apnoea: reflux-associated cough hypersensitivity? Eur Respir J. 2012;40(4):1049-50.

26. Yu Q, Liu Z, Liao J, Dan Z, Tian D. The characteristics of high-resolution esophageal manometry and multichannel intraluminal esophageal impedance and $\mathrm{pH}$ monitoring in gastroesophageal redlux cough. Chin J Gastroenterol Hepatol. 2015;24(10):1196-999.

27. Xavier SD, Moraes JP, Eckley CA. Prevalence of signs and symptoms of laryngopharyngeal reflux in snorers with suspected obstructive sleep apnea. Braz J Otorhinolaryngol. 2013;79:589-93. 
28. Laohasiriwong S, Johnston N, Woodson BT. Extra-esophageal reflux, nose score, and sleep quality in an adult clinic population. Laryngoscope. 2013;123:3233-8. doi:10.1002/lary.24236.

29. Shepherd KL, Holloway RH, Hillman DR, Eastwood PR. The impact of continuous positive airway pressure on the lower esophageal sphincter. Am J Physiol Gastrointest Liver Physiol 2007 292(5):G1200-5.

30. Shepherd K, Hillman D, Holloway R, Eastwood P. Mechanisms of nocturnal gastroesophageal reflux events in obstructive sleep apnea. Sleep Breath. 2011;15(3):561-70.

\section{Figures}

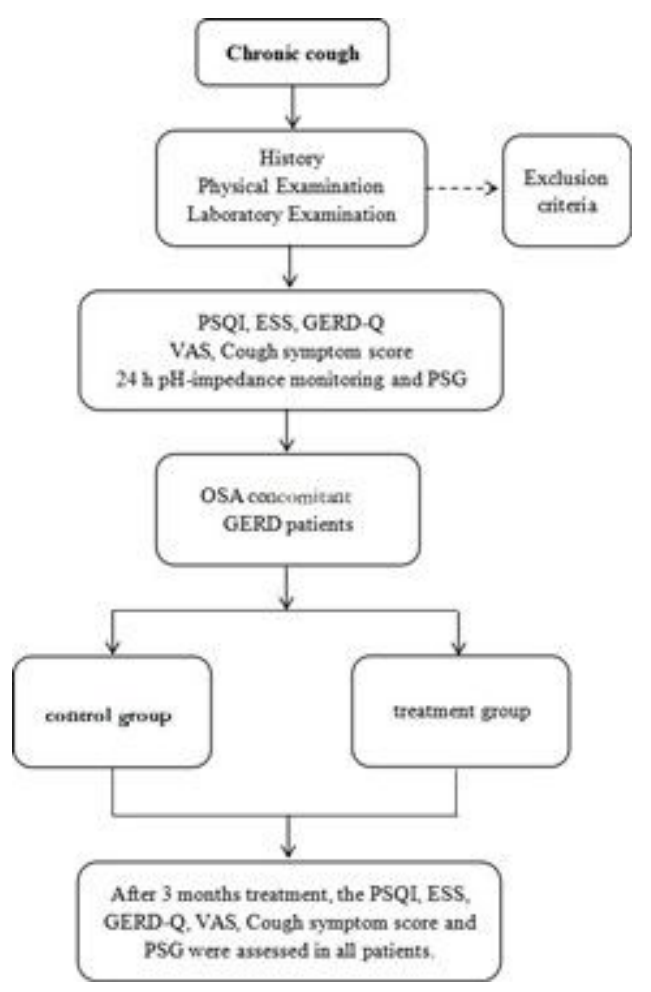

Figure 1

The protocol of this study.
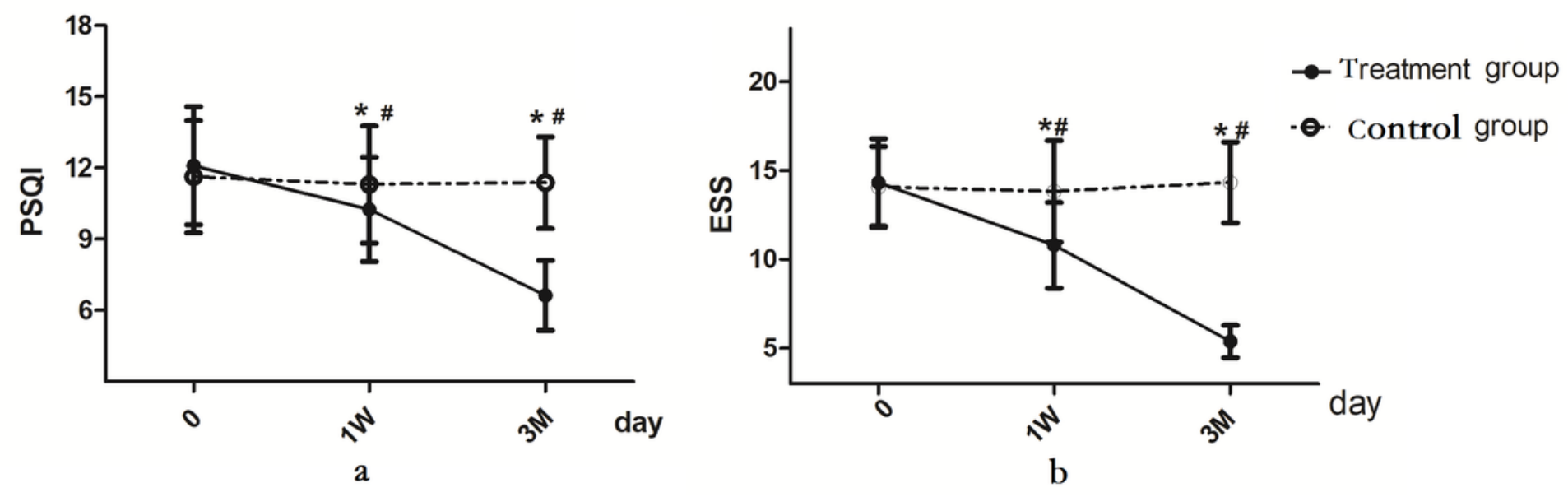
Figure 2

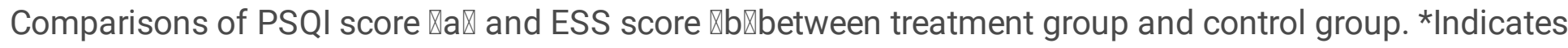
a significant difference between treatment group and control group at the same measurement time (P区 0.05). \#Indicates a significant improvement in treatment group when compared with the data of baseline $(P \llbracket 0.05)$.

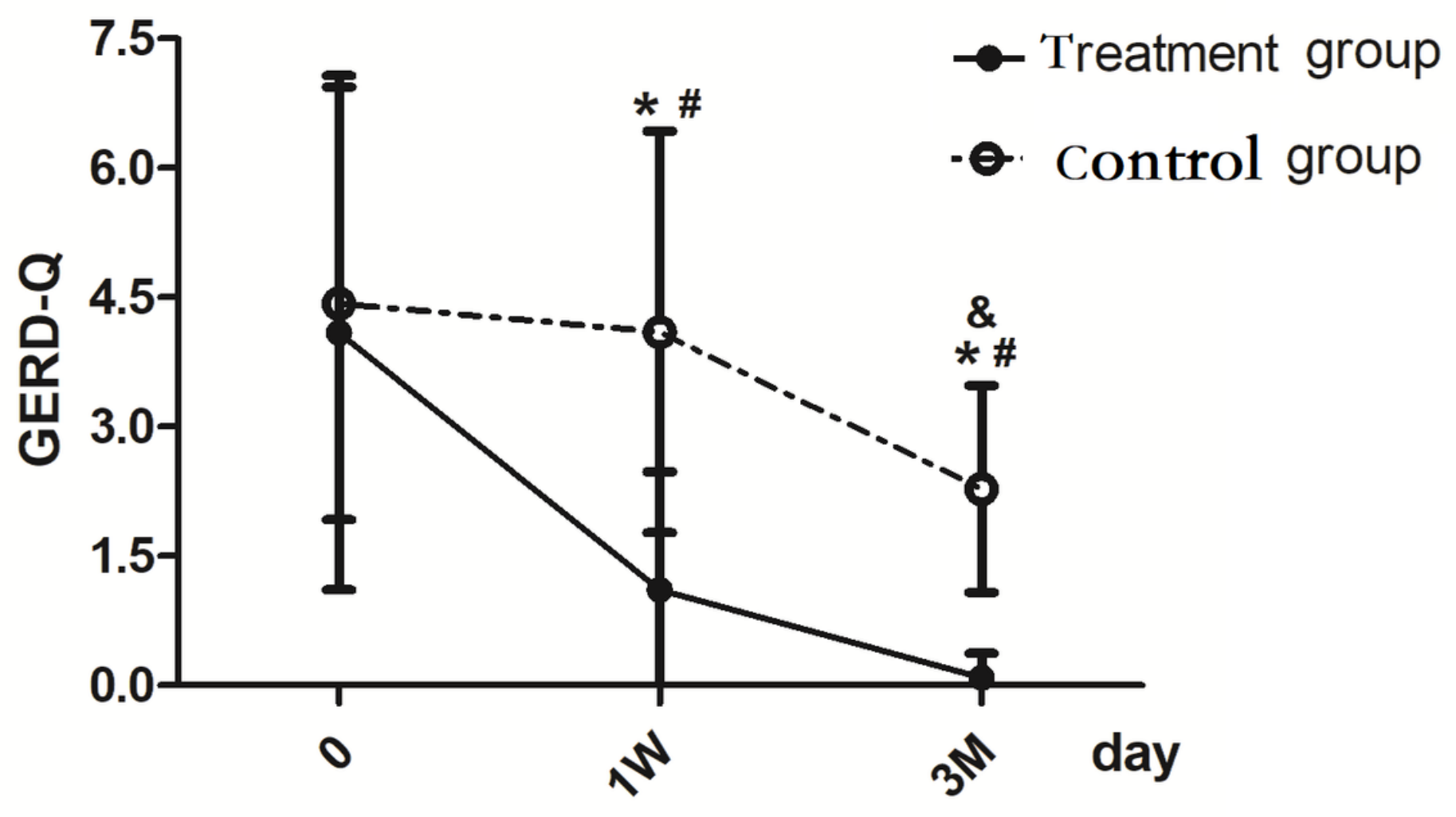

Figure 3

Comparisons of GERD-Q score between treatment group and control group. *Indicates a significant difference between treatment group and control group at the same measurement time ( $\mathrm{P} \otimes 0.05)$. \#Indicates a significant improvement in treatment group when compared with the data of baseline (P区 0.05). \&Indicates a significant improvement in control group when compared with the data of baseline (P区 $0.05)$. 

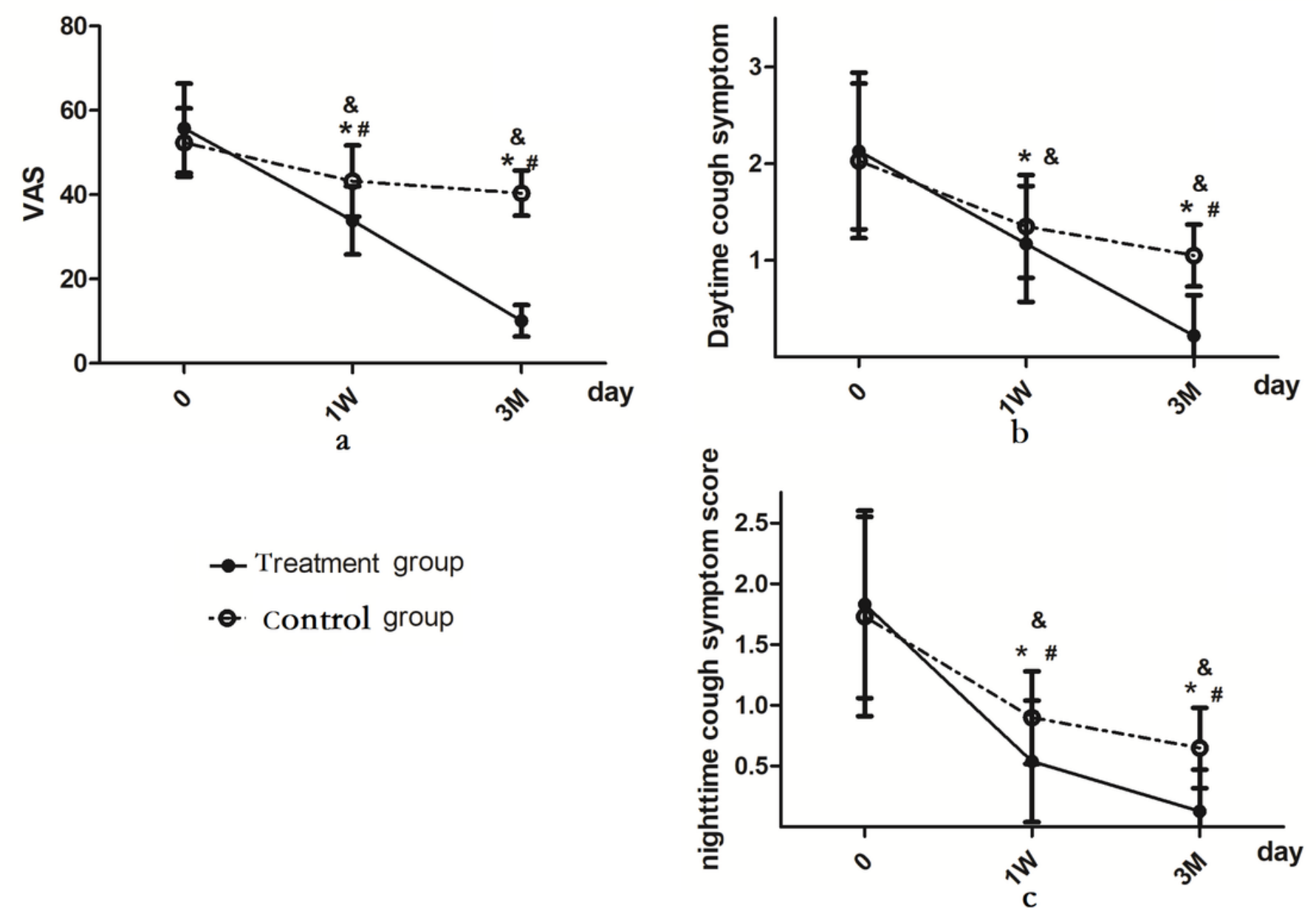

\section{Figure 4}

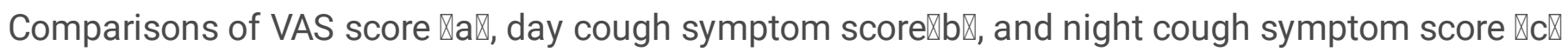
between treatment group and control group. *Indicates a significant difference between treatment group and control group at the same measurement time (P囚0.05). \#Indicates a significant improvement in treatment group when compared with the data of baseline (Pख0.05). \&Indicates a significant improvement in control group when compared with the data of baseline (P凶0.05). 

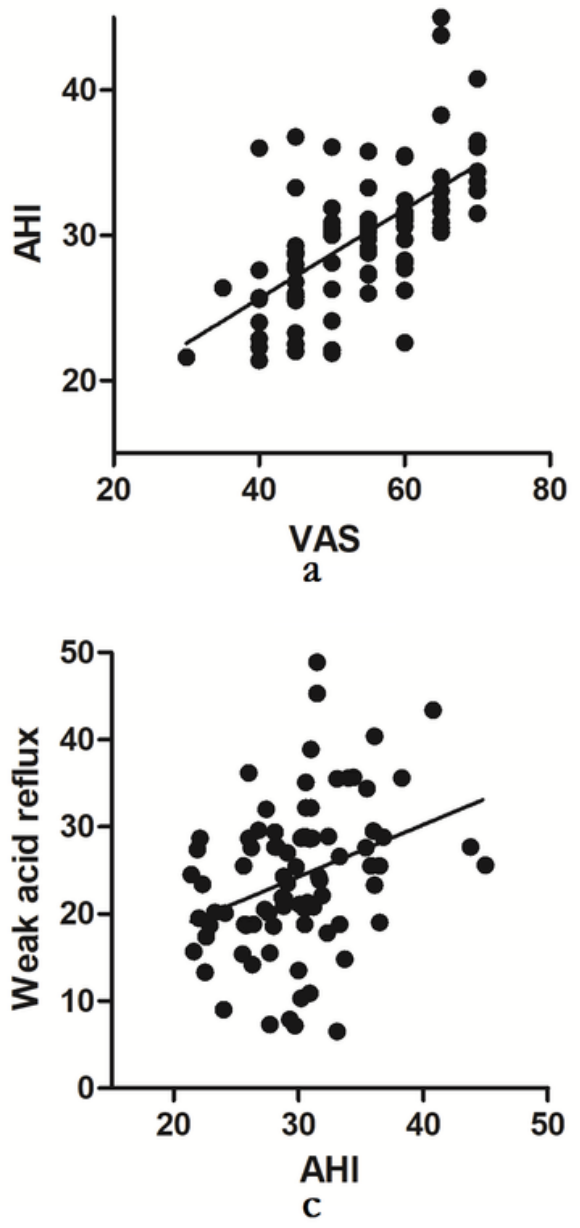

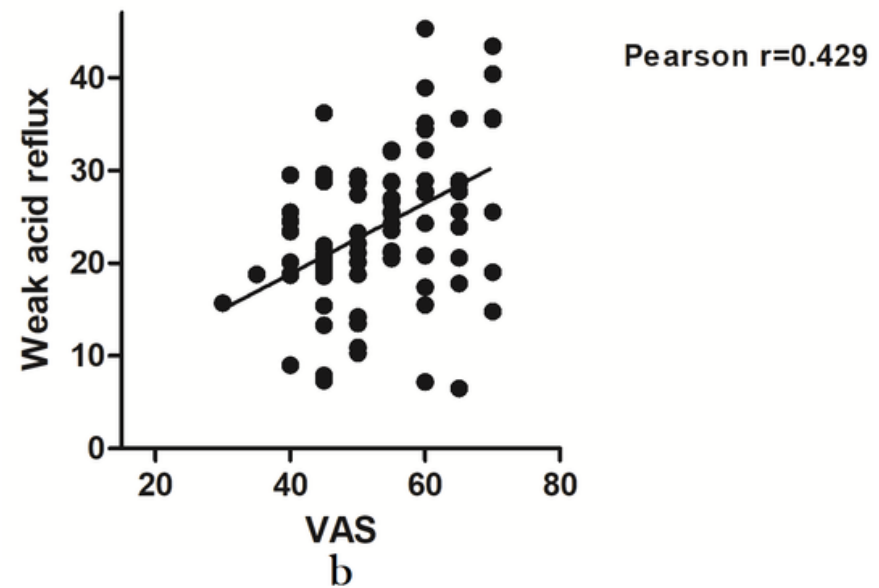

Pearson $r=0.366$

Figure 5

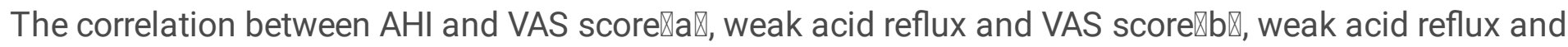
AHI囚C囚. 